\title{
Índex APGAR de Família na avaliação de relações familiares do idoso: revisão integrativa
}

\author{
Using the family APGAR score to evaluate family relationships in the elderly: an integrative review
}

Índice de APGAR de familia en la evaluación de relaciones familiares del anciano: revisión integrativa

Ivania Vera ${ }^{1}$, Roselma Lucchese ${ }^{2}$, Denize Bouttelet Munari ${ }^{3}$, Adélia Yaeko Kyosen Nakatani ${ }^{4}$

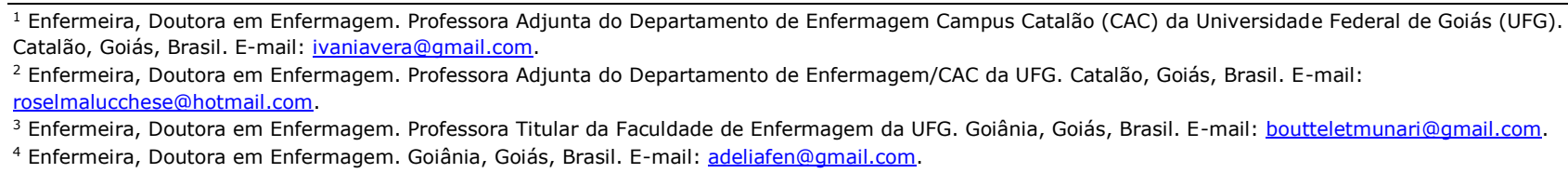

${ }^{4}$ Enfermeira, Doutora em Enfermagem. Goiânia, Goiás, Brasil. E-mail: adeliafen@gmail.com.

\section{RESUMO}

Revisão Integrativa da Literatura com o objetivo de sistematizar o conhecimento produzido acerca de como tem sido utilizado o APGAR de Família na identificação da funcionalidade nas relações familiares de idosos. Método: Levantamento realizado nas bases de dados da LILACS, MedLine, Pubmed e SciELO com os descritores: "aged", "aged, 80 and over", "family relations" e "family", por retratarem a tríade idoso-família-relações familiares nos idiomas português, inglês e espanhol, entre 2000 e 2013. Resultados: treze artigos atenderam aos critérios de inclusão, todos foram evidências de estudos não experimentais, descritivos ou com abordagem qualitativa. Os artigos analisados descrevem o APGAR de Família como um instrumento utilizado por equipe multiprofissional na avaliação da funcionalidade familiar a partir da pessoa índice. Rápido de ser aplicado, de fácil manejo profissional e interpretação, possibilita detecção de disfuncionalidade familiar de idosos e cuidadores, condição que colabora na ampliação da concepção das relações no contexto familiar.

Descritores: Idoso; Família; Relações Familiares; Enfermagem.

\section{ABSTRACT}

The objective of this Integrative Literature Review was to systematize the knowledge on how the Family APGAR score has been used to identify the functionality of family relationships in the elderly. Method: a was performed of LILACS, MedLine, Pubmed and SciELO databases using the descriptors: "aged", "aged, 80 and over", "family relations" and "family", as they represent the triad elderly-family-family relationships, published in Portuguese, English and Spanish between the year 2000 and 2013. Results: thirteen articles met the inclusion criteria, all of which were evidence of nonexperimental studies, descriptive or using a qualitative approach. The analyzed articles described the Family APGAR as an instrument used by a multidisciplinary team to measure family functionality based on the index person. The instrument is of quick application, easy to manage and interpret. Furthermore, it permits identifying family dysfunction of the elderly and caregivers, a condition that contributes to broadening the concept of relationships in the family setting. Descriptors: Aged; Family; Family Relations; Nursing.

\section{RESUMEN}

Revisión Integrativa de Literatura objetivando sistematizar el conocimiento producido sobre cómo ha sido utilizado el APGAR de Familia en la identificación de la funcionalidad en las relaciones familiares de ancianos. Método: Relevamiento realizado en bases de datos LILACS, MedLine, Pubmed y SciELO, con descriptores aged; aged, 80 and over; family relations y family, por retratar la tríada anciano-familia-relaciones familiares, en los idiomas portugués, inglés y español, entre 2000 y 2013. Resultados: Trece artículos cumplieron los criterios de inclusión. Todos fueron evidencias de estudios no experimentales, descriptivos o con abordaje cualitativo. Los artículos analizados describen el APGAR de Familia como un instrumento utilizado por equipo multiprofesional en evaluación de funcionalidad familiar a partir de la persona índice. De rápida aplicación, fácil manejo e interpretación profesional, posibilita detección de disfuncionalidad familiar de ancianos y cuidadores, condición que ayuda a ampliar la concepción de las relaciones en el contexto familiar.

Descriptores: Anciano; Familia; Relaciones Familiares; Enfermería. 


\section{INTRODUÇÃO}

Embora haja referências que procuram definir a família, vários fenômenos e contextos impedem uma determinação absoluta. Os novos arranjos familiares desvelam que na contemporaneidade, família possui inúmeras configurações ${ }^{(1)}$ e, estas recebem influência de grupos e classes sociais ${ }^{(2)}$. Assim, para se compreender o significado de família há de se considerar a contextualização destas variáveis.

Uma destas variáveis no âmbito sociodemográfico é o envelhecimento da população que imprime mudanças na composição familiar, tendo em vista que cada vez mais há presença de idosos na sociedade ${ }^{(3-4)}$. Além disso, a permanência da mulher como força de trabalho fora do ambiente doméstico, resulta na redução do apoio nuclear para 0 idoso(5-6). Tais alterações transformam as contribuições e atribuições dos diferentes componentes da família, mobilizando o convívio intergeracional ${ }^{(5,7-8)}$.

No entanto, a família pode apresentar dificuldades em aceitar e entender o envelhecimento de um de seus membros, o que, por vezes, torna a convivência familiar

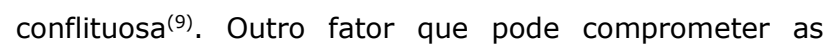
relações é a convivência com as limitações funcionais, muitas advindas de doenças crônicas e múltiplas, ocasionadas por enfermidades complexas e onerosas para a família(10).

Neste sentido, é importante que se trabalhe a capacidade funcional e autonomia do idoso, incentivando o envelhecimento ativo, na expectativa de mantê-lo independente, o que contribui na melhoria das relações familiares e sociais, para que se conquiste boa saúde adicionada aos anos de vida(11-12). Uma das formas de acompanhar esse processo é a avaliação da família, o que permite ao profissional de saúde conhecimento da sua funcionalidade e, por consequência, propor ações que fortaleçam a qualidade da atenção à pessoa idosa.

Uma ferramenta utilizada na identificação das relações e função familiar é o 'Family $A P G A R^{\prime}$, questionário desenvolvido por Gabriel Smilkstein, em 1978. Após ser traduzido, adaptado e validado, foi renomeado para 'APGAR de Família'(13-14).

Embora tenha sido idealizado para ser utilizado, exclusivamente, por médicos com propósito de fazer um diagnóstico da situação de vida de um novo paciente ou diante da necessidade de envolver a família no cuidado do idoso e na gestão de famílias ${ }^{(13)}$, também demonstrou capacidade de ajudar outros profissionais quando $o$ instrumento foi traduzido para a língua portuguesa e validado no Brasil(14).
O instrumento, que pode ser autoaplicado, permite a avaliação da satisfação subjetiva com o cuidado recebido do membro familiar por meio de cinco itens, a saber: Adaptation (Adaptação), Partnership (Companheirismo), Growth (Desenvolvimento), Affection (Afetividade) e Resolve (Capacidade resolutiva). A funcionalidade familiar (FF) é resultante do somatório dos valores obtidos (elevada disfuncionalidade -0 a 4; moderada disfuncionalidade - 5 e 6 ou boa funcionalidade - 7 a 10 pontos). Assim, a pontuação final possibilita a classificação dos níveis de funcionamento (disfuncionalidade e funcionalidade) familiar, sendo que o maior escore indica maior satisfação da pessoa índice que recebe o cuidado(13-14).

Uma família é considerada funcional quando há definição de tarefas ou funções claras e aceitas por seus membros familiares, no auxílio à resolução de problemas utilizando recursos próprios(15-16). Em situação oposta, a família disfuncional é aquela em que há desrespeito, sobreposição na hierarquia, ruídos na comunicação e não (re)organização do sistema familiar, na articulação de forças para resolução de problema ${ }^{(15-16)}$.

Uma breve revisão da literatura mostrou que a disfuncionalidade familiar foi associada à solidão em idosos $^{(17-18)}$, à falta de atenção e prestação de cuidados à familiares com Doenças Crônicas não Transmissíveis $(\mathrm{DCNT})^{(19)}$, à solidão de pessoas que vivem com HIV/AIDS $^{(20)}$ e ao restrito apoio social e sobrecarga de cuidadores de idosos ${ }^{(21)}$. Em sentido oposto, a FF por meio do APGAR de Família foi observada em situações de screening em candidatos à cirurgia bariátrica( ${ }^{(22)}$, em famílias convivendo e cuidando de idosos em diferentes situações de vulnerabilidade social(23), à melhor qualidade de vida de cuidadores de idosos ${ }^{(24)}$, residir em famílias nucleares e estar vinculado a atividade laboral ${ }^{(25)}$.

Diante dos resultados apontados com o uso da ferramenta do APGAR de Família considera-se fundamental que pesquisadores que a utilizam tenham mais clareza do seu alcance, isto é, que possam acessar as melhores evidências produzidas a partir do seu uso. Sendo assim, o objetivo deste estudo foi sistematizar o conhecimento produzido acerca de como tem sido utilizado o APGAR de Família na identificação da funcionalidade nas relações familiares de idosos.

\section{METODOLOGIA}

Revisão Integrativa de Literatura (RI) que se constitui em um método de revisão específica, a partir da organização da produção científica, em busca do estado da arte do assunto de interesse, com vista à avaliação 
crítica e síntese de evidências de outros estudos e metodologias (experimental, quase experimental, não experimental, qualitativo, quantitativo $)^{(26-28)}$. Método de pesquisa também utilizado para incorporar evidências da prática clínica junto ao cuidado ao indivíduo, família e comunidade prestada nos diversos níveis de atenção, em prol à prática baseada em evidência $(\mathrm{PBE})^{(28)}$. Para se realizar uma RI devem ser obedecidos os passos propostos na metodologia, tais como: identificação do tema e questão norteadora, definição dos critérios de inclusão e exclusão, definição das informações de interesse a serem extraídas dos textos selecionados, avaliação dos estudos, análise e interpretação dos resultados contidos nos textos e, apresentação da síntese do conhecimento ${ }^{(26-28)}$. A questão que norteou a presente investigação foi "Qual a contribuição das publicações que utilizaram o índex APGAR de Família na identificação do fenômeno da funcionalidade familiar nas famílias com idosos?"

O levantamento foi realizado nas bases de dados da Literatura Latino-Americana e do Caribe em Ciências da Saúde (LILACS); Medical Literature Analysis and Retrieval Sistem on line (MedLine), Pubmed e Scientific Electronic Library Online (SciELO). A busca foi efetuada no mês de dezembro de 2011 e setembro de 2013 por dois pesquisadores de forma independente.

Para a busca selecionou-se os descritores controlados extraídos dos Descritores em Ciências da Saúde (DeCS) para indexação de artigos e do Medical Subject Headings (Mesh) da National Library: "aged", "aged, 80 and over", "family relations" e "family". Estes foram escolhidos por retratarem a tríade idoso-família-relações familiares, uma vez que o acrônimo APGAR de Família não é um descritor controlado.

Os critérios de inclusão das publicações desta RI foram: apenas artigos científicos, com textos completos originais, disponíveis eletronicamente e via Comutação Bibliográfica (COMUT) que abordassem o relacionamento do idoso com a família, publicados a partir de janeiro de 2000 à setembro de 2013 disponíveis em idioma na língua inglesa, espanhola e portuguesa e que utilizaram o índex APGAR de Família. Foram excluídos os artigos que se repetiram nas bases de dados.

Para cada artigo da amostra final deste estudo, foi preenchido o formulário adaptado e proposto por Ursi(29), após tradução e leitura exaustiva. As informações de interesse retiradas foram: titulo do artigo, autores, ano de publicação, país de origem, base de dados, amostra, delineamento do estudo, instrumentos e índex utilizados pelos pesquisadores na pesquisa original, síntese das conclusões e principais resultados encontrados e nível de evidência científica. Para análise do nível de evidência se utilizou, a classificação hierárquica proposta por Stetler et al. ${ }^{(30)}$ : nível I - evidência obtida do resultado de metanálise de estudos clínicos controlados e com randomização; nível II - evidência obtida em estudo de desenho experimental; nível III - evidência obtida de pesquisas quase experimentais; nível IV- evidências obtidas de estudos não experimental, descritivos ou com abordagem metodológica qualitativa; nível V- evidências obtidas de relatórios de casos ou relatos de experiências; nível VI- evidências baseadas em opiniões de especialistas ou com base em normas ou legislação.

A Figura 1 revela os passos percorridos por esta RI. A partir dos artigos que preencheram os critérios de inclusão pré-estabelecidos houve leitura e extração das informações de interesse. Foi realizada também uma busca manual (hand search) como estratégia de investigação nas listas da bibliografia e referências dos artigos originais com intuito de maior exploração das publicações $^{(28)}$. 


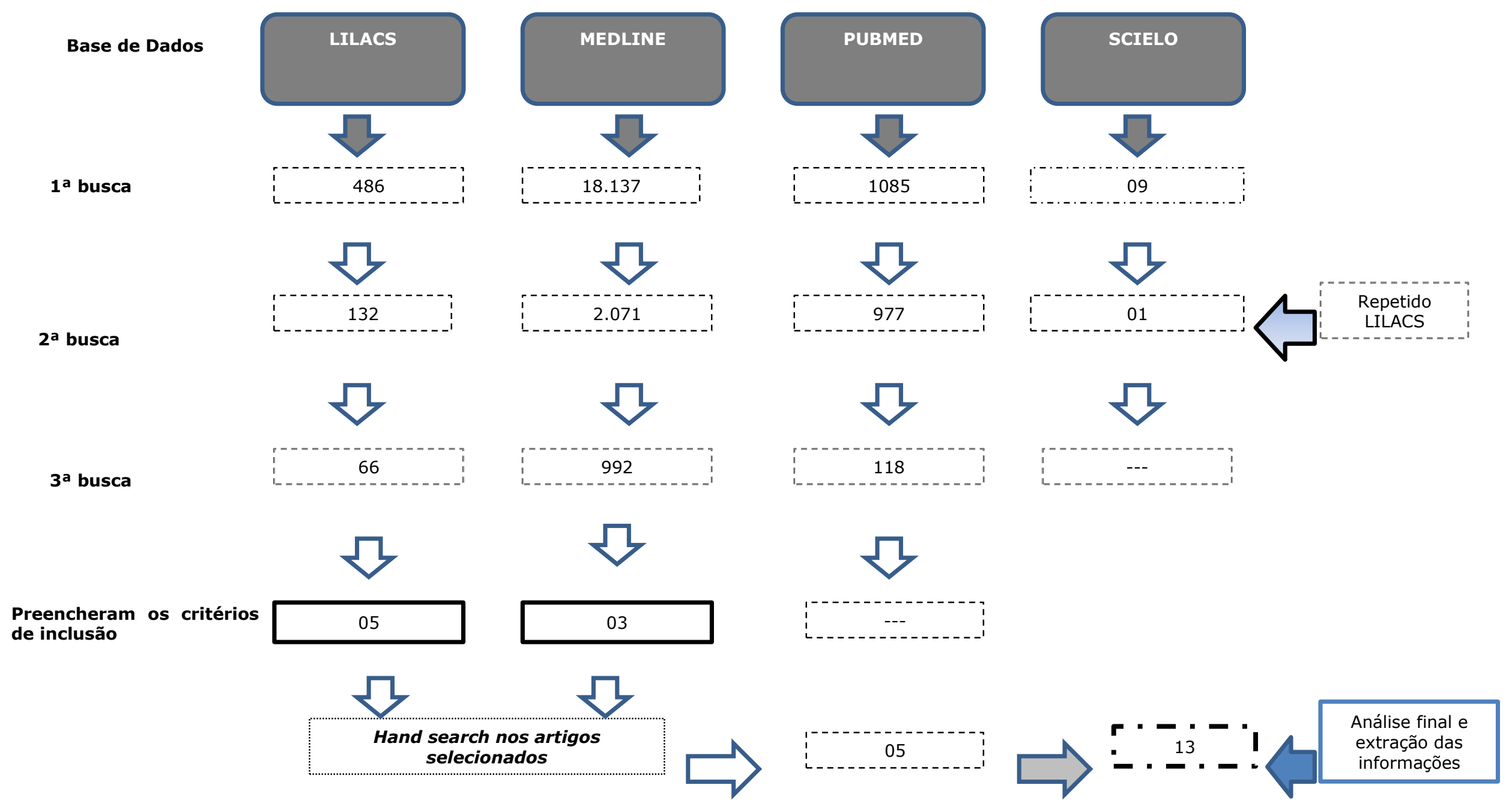

Figura 1: Diagrama de fluxo do processo de seleção dos artigos da amostra, 2000-2013. 


\section{RESULTADOS}

A amostra desta RI resultou em cinco artigos na LILACS e três na MedLine. A partir destes textos, na hand search, foram incluídos mais quatro artigos da LILACS e um da PubMed, totalizando em treze artigos para análise final. Em números percentuais, $69,2 \%$ dos artigos estavam na LILACS, 23,1\% na MedLine e 7,7\% na Pubmed. Na intenção de sumarizar, integralizar os achados desta RI e destacar as contribuições do APGAR de Família na compreensão das relações familiares foi construído um quadro síntese (Quadro 1).

Quadro 1: Síntese dos artigos que utilizaram o Índex APGAR de Família. 2000-2013.

\begin{tabular}{|c|c|c|c|c|c|}
\hline Título & Autores & $\begin{array}{c}\text { Ano / } \\
\text { País / } \\
\text { Base de } \\
\text { dados / } \\
\text { Amostra }\end{array}$ & $\begin{array}{c}\text { Delineamento do } \\
\text { estudo / Nível de } \\
\text { evidência / } \\
\text { Instrumentos }\end{array}$ & $\begin{array}{l}\text { Síntese das } \\
\text { conclusões }\end{array}$ & Resultados \\
\hline $\begin{array}{c}\text { Percepção de } \\
\text { idosos pobres com } \\
\text { alterações } \\
\text { cognitivas sobre } \\
\text { funcionalidade } \\
\text { familiar(23). }\end{array}$ & $\begin{array}{c}\text { Santos AA; } \\
\text { Pavarini SCI; } \\
\text { Barham EJ. }\end{array}$ & $\begin{array}{c}2011 \text { / } \\
\text { Brasil / } \\
\text { LILACS / } \\
45 \text { idosos }\end{array}$ & $\begin{array}{l}\text {-Descritivo, } \\
\text { transversal (NÍVEL } \\
\text { IV) } \\
\text {-APGAR Smilkstein. }\end{array}$ & $\begin{array}{l}\text { - apenas as mulheres } \\
\text { apresentaram } \\
\text { elevada disfunção } \\
\text { familiar, perfazendo } \\
17 \% \text {. }\end{array}$ & $\begin{array}{l}\text {-Não houve } \\
\text { correlação } \\
\text { significativa entre a } \\
\text { idade e o APGAR; } \\
\text {-Há lacunas na } \\
\text { literatura sobre a } \\
\text { funcionalidade } \\
\text { familiar de idosos. }\end{array}$ \\
\hline $\begin{array}{l}\text { Relationships } \\
\text { between quality of } \\
\text { life and family } \\
\text { function in } \\
\text { caregiver }^{(24)} \text {. }\end{array}$ & $\begin{array}{c}\text { Rodríguez- } \\
\text { Sánchez E; } \\
\text { Pérez- } \\
\text { Peñaranda A; } \\
\text { Losada-Baltar } \\
\text { A; Pérez- } \\
\text { Arechaederra } \\
\text { D; Gómez- } \\
\text { Marcos MÁ; } \\
\text { Patino-Alonso } \\
\text { M; García-Ortiz } \\
\text { L. }\end{array}$ & $\begin{array}{c}2011 \text { / } \\
\text { Espanha / } \\
\text { MEDLINE / } \\
153 \\
\text { caregivers }\end{array}$ & $\begin{array}{l}\text {-Cross-sectional } \\
\text { study (NÍVEL IV) } \\
\text {-APGAR Smilkstein; } \\
\text {-QoL (Ruiz-Baca-Q) } \\
\text {-The Canonical } \\
\text { correspondence } \\
\text { Analysis (CCA) }\end{array}$ & $\begin{array}{l}\text { - } 69.93 \% \text { of the } \\
\text { families were in a } \\
\text { situation of normal } \\
\text { functionality. }\end{array}$ & $\begin{array}{l}\text {-Association } \\
\text { between family } \\
\text { functionality and the } \\
\text { caregiver's QoL. }\end{array}$ \\
\hline $\begin{array}{c}\text { Valores y } \\
\text { funcionabilidad } \\
\text { familiar em } \\
\text { habitantes del } \\
\text { sector San Jose } \\
\text { Coro-Falcón(31). }\end{array}$ & Ferrer M. & $\begin{array}{c}2003 \text { / } \\
\text { Venezuela } \\
\text { / LILACS / } \\
95 \text { familias }\end{array}$ & $\begin{array}{l}\text {-Descriptivo, } \\
\text { correlacional, não } \\
\text { experimental, } \\
\text { transeccional de } \\
\text { campo (NÍVEL IV) } \\
\text {-Teste de Garcia } \\
\text { Hos; } \\
\text {-APGAR Smilkstein. }\end{array}$ & $\begin{array}{l}\text {-Valores corporales, } \\
\text { intelectuales, } \\
\text { afectivos, } \\
\text { individuales, morales, } \\
\text { sociales, ecológicos y } \\
\text { religiosos se } \\
\text { relacionam } \\
\text { significativamente co } \\
\text { la funcionabilidad } \\
\text { familiar. } \\
\text {-75\%: buena función } \\
\text { familiar; }\end{array}$ & $\begin{array}{l}\text {-No se encuentran } \\
\text { evidencias } \\
\text { significativas que } \\
\text { haya relación entre } \\
\text { los valores estéticos } \\
\text { e instrumentais y la } \\
\text { funcionabilidad } \\
\text { familiar. }\end{array}$ \\
\hline
\end{tabular}




\begin{tabular}{|c|c|c|c|c|c|}
\hline Título & Autores & $\begin{array}{l}\text { Ano / } \\
\text { País / } \\
\text { Base de } \\
\text { dados / } \\
\text { Amostra }\end{array}$ & $\begin{array}{c}\text { Delineamento do } \\
\text { estudo / Nível de } \\
\text { evidência / } \\
\text { Instrumentos }\end{array}$ & $\begin{array}{l}\text { Síntese das } \\
\text { conclusões }\end{array}$ & Resultados \\
\hline $\begin{array}{l}\text { Factores de estrés } \\
\qquad \text { y apoyo } \\
\text { psicosocial en } \\
\text { pacientes con } \\
\text { infarto agudo de } \\
\text { miocardio. Cali, } \\
2001-2002^{(32)} \text {. }\end{array}$ & $\begin{array}{l}\text { Reyes CA; } \\
\text { Hincapié M; } \\
\text { Herrera MJA; } \\
\text { Moyano PA. }\end{array}$ & $\begin{array}{c}2004 \text { / } \\
\text { Colômbia / } \\
\text { LILACS / } \\
78 \\
\text { personas }\end{array}$ & $\begin{array}{l}\text {-Descriptivo con } \\
\text { grupo control (NÍVEL } \\
\text { IV) } \\
\text {-Teste de Depressão } \\
\text { Geriátrica de } \\
\text { Yasavage (GDS); } \\
\text {-APGAR Smilkstein; } \\
\text {-Escala de Holmes e } \\
\text { Rahe }\end{array}$ & $\begin{array}{l}\text {-La disfunción } \\
\text { familiar, la depresión } \\
\text { y los eventos de } \\
\text { cambio de vida no } \\
\text { mostraron } \\
\text { diferenciais entre los } \\
\text { grupos; } \\
\text {-40\%: Disfunción } \\
\text { familiar leve }\end{array}$ & $\begin{array}{l}\text { - No se observaron } \\
\text { diferencias entre los } \\
\text { grupos los aspectos } \\
\text { psicosociales. } \\
\text {-La práctica religiosa } \\
\text { puede ser un } \\
\text { recurso de apoyo } \\
\text { importante para la } \\
\text { salud de los } \\
\text { pacientes ansianos. }\end{array}$ \\
\hline $\begin{array}{c}\text { Funcionamiento } \\
\text { social del adulto } \\
\text { mayor(33). }^{(3)}\end{array}$ & $\begin{array}{l}\text { Zavala GM; } \\
\text { Vidal GD; } \\
\text { Castro SM; } \\
\text { Quiroga P; } \\
\text { Gonzalo KP. }\end{array}$ & $\begin{array}{c}2006 \text { / } \\
\text { Chile / } \\
\text { LILACS / } \\
300 \\
\text { ancianos y } \\
\text { sus } \\
\text { cuidadores }\end{array}$ & $\begin{array}{l}\text {-Estudio descriptive } \\
\text { (NÍVEL IV) } \\
\text {-Groningen Social } \\
\text { Disabilities Schedule; } \\
\text {-APGAR Smilkstein; } \\
\text {-Test de Autoestima } \\
\text { de Rosenberg }\end{array}$ & $\begin{array}{l}\text {-Funcionamiento } \\
\text { familiar en } 84 \% \text {. }\end{array}$ & $\begin{array}{l}\text {-No se encontró } \\
\text { diferencia } \\
\text { estadísticamente } \\
\text { significativa en la } \\
\text { evaluación de la } \\
\text { percepción de } \\
\text { funcionamiento } \\
\text { social y } \\
\text { autoevaluación. }\end{array}$ \\
\hline $\begin{array}{c}\text { Quem irá } \\
\text { empurrar minha } \\
\text { cadeira de rodas? } \\
\text { A escolha do } \\
\text { cuidador familiar } \\
\text { do idoso } \\
\text { (34). }\end{array}$ & $\begin{array}{l}\text { Pavarini SCI; } \\
\text { Tonon FL; } \\
\text { Silva JMC; } \\
\text { Mendiondo } \\
\text { MSZ; Barham } \\
\text { EJ; Filizola } \\
\text { CLA. }\end{array}$ & $\begin{array}{c}2006 \text { / } \\
\text { Brasil / } \\
\text { LILACS / } \\
93 \text { idosos }\end{array}$ & $\begin{array}{l}\text {-Descritivo } \\
\text { transversal (NÍVEL } \\
\text { IV) } \\
\text {-Análise Conteúdo- } \\
\text { Bardin; } \\
\text {-APGAR Smilkstein; }\end{array}$ & $\begin{array}{l}\text { - A escolha por um } \\
\text { cuidador é } \\
\text { multideterminada, } \\
\text { multivariada e se } \\
\text { processa ao longo da } \\
\text { vida. } \\
\text {-87,1\%: boa } \\
\text { funcionalidade } \\
\text { Familiar. }\end{array}$ & $\begin{array}{l}\text {-Permite a } \\
\text { compreensão da } \\
\text { dinâmica familiar e } \\
\text { dos relatos dos } \\
\text { entrevistados sobre } \\
\text { a escolha do seu } \\
\text { cuidador na velhice. }\end{array}$ \\
\hline $\begin{array}{l}\text { Funcionalidade } \\
\text { familiar de idosos } \\
\text { dependentes } \\
\text { residentes em } \\
\text { domicílios(35). }\end{array}$ & $\begin{array}{c}\text { Torres GV; } \\
\text { Reis LA; Reis } \\
\text { LA; Fernandes } \\
\text { MH; Alves GS, } \\
\text { Sampaio LS; } \\
\text { Mascarenhas } \\
\text { CHM. }\end{array}$ & $\begin{array}{c}2009 \text { / } \\
\text { Brasil / } \\
\text { LILACS / } \\
117 \text { idosos }\end{array}$ & $\begin{array}{l}\text {-Pesquisa de caráter } \\
\text { analítica; transversal } \\
\text { (NÍVEL IV) } \\
\text {-Índice de Barthel } \\
\text {-APGAR Smilkstein }\end{array}$ & $\begin{array}{l}-73,5 \% \text { : } \\
\text { disfuncionalidade } \\
\text { familiar para idosos } \\
\text { dependentes. }\end{array}$ & $\begin{array}{l}\text {-Muitas famílias não } \\
\text { estão preparadas } \\
\text { para prestar } \\
\text { cuidados aos idosos } \\
\text { portadores de } \\
\text { problemas de saúde. }\end{array}$ \\
\hline $\begin{array}{l}\text { Relação entre } \\
\text { funcionalidade } \\
\text { familiar e } \\
\text { capacidade } \\
\text { funcional de } \\
\text { idosos } \\
\text { dependentes no } \\
\text { município de } \\
\text { Jequié }(B A)^{(36)} \text {. }\end{array}$ & $\begin{array}{c}\text { Torres GV; } \\
\text { Reis LA; Reis } \\
\text { LA; Fernandes } \\
\text { MH; Xavier TT. }\end{array}$ & $\begin{array}{c}2010 \text { / } \\
\text { Brasil / } \\
\text { LILACS / } \\
117 \text { idosos }\end{array}$ & $\begin{array}{l}\text {-Analítico/transversal } \\
\text { (NÍVEL IV) } \\
\text {-APGAR Smilkstein; } \\
\text {-WHOQOL-OLD; } \\
\text {-Índice de Barthel. }\end{array}$ & $\begin{array}{l}\text {-73,5\% dos idosos } \\
\text { relataram } \\
\text { comprometimento da } \\
\text { dinâmica familiar. }\end{array}$ & $\begin{array}{l}\text {-Não foi encontrada } \\
\text { diferença estatística } \\
\text { entre } \\
\text { comprometimento } \\
\text { da dinâmica familiar } \\
\text { e capacidade } \\
\text { funcional do idoso } \\
\text { dependente. }\end{array}$ \\
\hline
\end{tabular}




\begin{tabular}{|c|c|c|c|c|c|}
\hline Título & Autores & $\begin{array}{l}\text { Ano / } \\
\text { País / } \\
\text { Base de } \\
\text { dados / } \\
\text { Amostra }\end{array}$ & $\begin{array}{c}\text { Delineamento do } \\
\text { estudo / Nível de } \\
\text { evidência / } \\
\text { Instrumentos }\end{array}$ & $\begin{array}{l}\text { Síntese das } \\
\text { conclusões }\end{array}$ & Resultados \\
\hline $\begin{array}{l}\text { Family function } \\
\text { and the mental } \\
\text { health of the } \\
\text { caregiver of } \\
\text { dependent } \\
\text { relatives }{ }^{(37)} \text {. }\end{array}$ & $\begin{array}{c}\text { Perez- } \\
\text { Penaranda A; } \\
\text { Garcia Ortiz L; } \\
\text { Rodriguez- } \\
\text { Sanchez E; } \\
\text { Losada Baltar } \\
\text { A; Porras } \\
\text { Santos N; } \\
\text { Gomez Marcos } \\
\text { MA. }\end{array}$ & $\begin{array}{c}2009 \text { / } \\
\text { Espanha / } \\
\text { PUBMED / } \\
153 \\
\text { caregivers }\end{array}$ & $\begin{array}{l}\text { - Cross-sectional } \\
\text { study (NÍVEL IV) } \\
\text {-General Health } \\
\text { Quest. (GHQ-12) } \\
\text {-APGAR Smilkstein; } \\
\text {-Duke University } \\
\text { - Pfeiffer and } \\
\text { Barthel. }\end{array}$ & $\begin{array}{l}\text {-La función familiar } \\
\text { es um importante } \\
\text { predictor de la salud } \\
\text { mental de los } \\
\text { cuidadores de } \\
\text { pacientes } \\
\text { dependientes. } \\
-69,7 \% \text { : } \\
\text { normofuncionalidad } \\
\text { familiar; }\end{array}$ & $\begin{array}{l}\text {-Las variables } \\
\text { asociadas fueron el } \\
\text { consumo de } \\
\text { psicofarmacos, la } \\
\text { disfuncionalidad } \\
\text { familiar, el escaso } \\
\text { apoyo social y el } \\
\text { bajo nível educativo. }\end{array}$ \\
\hline $\begin{array}{c}\text { Sobrecarga } \\
\text { asociada con el } \\
\text { cuidado de } \\
\text { ancianos } \\
\text { dependientes }^{(38)} \text {. }\end{array}$ & $\begin{array}{l}\text { Ocampo JM; } \\
\text { Herrera JÁ; } \\
\text { Torres P; } \\
\text { Rodríguez JA; } \\
\text { Loboa L; } \\
\text { García CA. }\end{array}$ & $\begin{array}{c}2007 \text { / } \\
\text { Colômbia / } \\
\text { LILACS / } \\
35 \\
\text { ancianos }\end{array}$ & $\begin{array}{l}\text { - Comparativo, } \\
\text { observacional, de } \\
\text { corte transversal } \\
\text { (NÍVEL IV) } \\
\text {-Índice de Barthel, } \\
\text { Escala de Zarit } \\
\text {-Minimental (MEEM) } \\
\text {-APGAR Smilkstein } \\
\text {-Escala de Depressão } \\
\text { Geriátrica de } \\
\text { Yesavage }\end{array}$ & $\begin{array}{l}\text {-54.2\% de los } \\
\text { cuidadores no había } \\
\text { sobrecarga; } \\
\text {-ancianos } \\
\text { dependientes en las } \\
\text { actividades básicas } \\
\text { cotidianas en el } \\
\text { aspecto físico se } \\
\text { asociaron con la } \\
\text { carga percibida por el } \\
\text { cuidador }\end{array}$ & $\begin{array}{l}\text {-asociaciones } \\
\text { estadísticas entre } \\
\text { Minimental, } \\
\text { depresión geriátrica } \\
\text { y APGAR familiar } \\
\text { entre la presencia } \\
\text { de sobrecarga para } \\
\text { el cuidador con } \\
\text { número de } \\
\text { enfermedades } \\
\text { crônicas; }\end{array}$ \\
\hline $\begin{array}{l}\text { Funcionalidade } \\
\text { familiar de idosos } \\
\text { com alterações } \\
\text { cognitivas: a } \\
\text { percepção do } \\
\text { cuidador(39). }\end{array}$ & $\begin{array}{c}\text { Santos AA; } \\
\text { Pavarini SCI. }\end{array}$ & $\begin{array}{c}2012 \text { / } \\
\text { Brasil / } \\
\text { MEDLINE / } \\
72 \\
\text { cuidadores }\end{array}$ & $\begin{array}{l}\text {-descritivo, } \\
\text { transversal, baseado } \\
\text { nos pressupostos da } \\
\text { pesquisa } \\
\text { quantitativa; (NÍVEL } \\
\text { IV) } \\
\text {-Índice Paulista de } \\
\text { Vulnerabilidade } \\
\text { Social (IPVS) } \\
\text {-Minimental (MEEM) } \\
\text {-APGAR de Família }\end{array}$ & $\begin{array}{l}\text {-82\% dos cuidadores } \\
\text { com BFF; } \\
-43 \% \text { dos cuidadores } \\
\text { tem ensino } \\
\text { fundamental; } \\
-83 \% \text { dos cuidadores } \\
\text { moram em contexto } \\
\text { de pobreza }\end{array}$ & $\begin{array}{l}\text {-correlação } \\
\text { estatisticamente } \\
\text { significativa entre } \\
\text { APGAR de Família e } \\
\text { número de } \\
\text { moradores no } \\
\text { domicílio }\end{array}$ \\
\hline $\begin{array}{c}\text { Correlation } \\
\text { between Family } \\
\text { APGAR scores and } \\
\text { health-related } \\
\text { quality of life of } \\
\text { Filipino elderly } \\
\text { patients with knee } \\
\text { osteoarthritis(40). }\end{array}$ & $\begin{array}{c}\text { Lim ATC; } \\
\text { Manching J; } \\
\text { Penserga EG. }\end{array}$ & $\begin{array}{c}2012 \text { / } \\
\text { Filipinas / } \\
\text { MEDLINE / } \\
90 \text { patients }\end{array}$ & $\begin{array}{l}\text {-cross-sectional, } \\
\text { analytical (NÍVEL IV) } \\
\text {-Western Ontario } \\
\text { and McMaster } \\
\text { Universities } \\
\text { Osteoarthritis Index } \\
\text { (WOMAC); } \\
\text {-Family APGAR } \\
\text { - HrQoL }\end{array}$ & $\begin{array}{l}-53 \% \text { BFF } \\
\text {-that higher Family } \\
\text { APGAR score } \\
\text { correlated with better } \\
\text { HrQol }\end{array}$ & $\begin{array}{l}\text {-concludes that } \\
\text { higher Family } \\
\text { APGAR scores in this } \\
\text { population } \\
\text { correlated with } \\
\text { better HrQoL. }\end{array}$ \\
\hline
\end{tabular}




\begin{tabular}{|c|c|c|c|c|c|}
\hline Título & Autores & $\begin{array}{c}\text { Ano / } \\
\text { País / } \\
\text { Base de } \\
\text { dados / } \\
\text { Amostra }\end{array}$ & $\begin{array}{c}\text { Delineamento do } \\
\text { estudo / Nível de } \\
\text { evidência / } \\
\text { Instrumentos }\end{array}$ & $\begin{array}{l}\text { Síntese das } \\
\text { conclusões }\end{array}$ & Resultados \\
\hline $\begin{array}{l}\text { Convívio e cuidado } \\
\text { familiar na quarta } \\
\text { idade: qualidade } \\
\text { de vida de idosos } \\
\text { e seus } \\
\text { cuidadores(41). }\end{array}$ & $\begin{array}{l}\text { Gonçalves } \\
\text { LTH; Leite MT; } \\
\text { Hildebrandt } \\
\text { LM; Bisogno } \\
\text { SC; Biasuz S; } \\
\text { Falcade BL. }\end{array}$ & $\begin{array}{c}2013 \text { / } \\
\text { Brasil / } \\
\text { LILACS / } \\
100 \text { idosos } \\
\text { e seus } \\
\text { cuidadores }\end{array}$ & $\begin{array}{l}\text {-descritivo (NÍVEL } \\
\text { IV); } \\
\text {-APGAR de Família } \\
\text {-Whoqol-Old, } \\
\text {-Whoqol-Breve } \\
\text {-Estilo de vida de } \\
\text { Nahas }\end{array}$ & $\begin{array}{l}\text {-80\% dos idosos com } \\
\text { EDF; } \\
\text {-WHOQOL-Breve - } \\
\text { média dos } \\
\text { cuidadores: } 84,15 \\
\text {-WHOQOL-OId - } \\
\text { média dos idosos: } \\
78,48 \\
\text {-Escala de Nahas: } \\
\text { escore médio de } \\
23,89\end{array}$ & $\begin{array}{l}\text { - o convívio } \\
\text { doméstico e } \\
\text { cuidados na quarta } \\
\text { idade afetam a FF e } \\
\text { reflete na QV e } \\
\text { estilo de vida dos } \\
\text { cuidadores; } \\
\text {-deve-se } \\
\text { redirecionar as } \\
\text { políticas públicas e } \\
\text { sociais a favor da } \\
\text { rede de apoio à } \\
\text { população idosa } \\
\text { mais envelhecida, } \\
\text { extensiva às famílias } \\
\text { cuidadoras. }\end{array}$ \\
\hline
\end{tabular}

Na caracterização dos artigos estudados, encontrouse a utilização do APGAR de Família em grupos heterogêneos em relação ao gênero, capacidade funcional, comorbidades, função cognitiva dos idosos.

Estes estudos permeiam desde a identificação precoce da disfuncionalidade familiar em famílias ${ }^{(31)}$, pacientes com doenças agudas( ${ }^{(32)}$ e crônicas ${ }^{(40)}$. Houve um aumento progressivo de estudos na identificação precoce da FF desde a escolha do cuidador ${ }^{(34)}$, de cuidadores de pessoas idosas com enfermidades crônicas ${ }^{(33,36,40)}$, sobrecarga

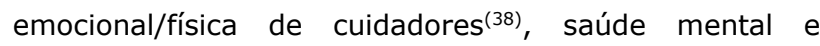
qualidade de vida de cuidadores ${ }^{(24,37,41)}$, idosos com função cognitiva comprometida ${ }^{(23)}$, idosos dependentes ${ }^{(35-36)}$, bem como, a influência da FF na quantidade de moradores que residem em um mesmo domicílio(39,41). Todas estas situações encontradas colaboram na ampliação da concepção das relações no contexto familiar em que o idoso está inserido.

Quanto à origem dos estudos observa-se que $77 \%$ (10) são provenientes da América Latina, 15,4\% da Europa (02) e 7,7\% da Ásia (01). Em relação ao tipo de revista científica, houve predomínio na publicação internacional com sete artigos $(53,8 \%)$ e seis artigos $(46,2 \%)$ nacionais. Em $100 \%$ dos estudos houve abordagem qualitativa, descritiva, com amostra de conveniência e delineamento não experimental, portanto caracterizando nível IV de evidência de acordo com a classificação hierárquica proposta por Stetler et al(30).
A utilização do índex foi multiprofissional, prevalecendo enfermeiros, seguidos por médicos, fisioterapeuta, assistente social e psicólogo. Não houve publicações nos anos 2000, 2001, 2002, 2005 e 2008, de acordo com os critérios de inclusão estabelecidos nesta RI.

Todos os artigos analisados descrevem que o APGAR de Família é um instrumento de avaliação da FF de fácil manejo profissional e interpretação, rápido na aplicação, além de possibilitar a detecção precoce da disfunção familiar. Houve relevância no aspecto de favorecer a concepção do sujeito índice quanto à qualidade da relação familiar.

\section{DISCUSSÃO}

Quanto à origem e o meio de circulação, a maioria das publicações pertence à América do Sul, o que reflete e compreensão da realidade sul americana na percepção da funcionalidade familiar ${ }^{(23,31-36,38-39,41)}$. Mesmo aqueles que foram divulgados em revistas de meio europeu receberam contribuição de autores latino-americanos ao avaliaram a percepção do cuidador de idosos com sua família(24,37).

Este aspecto reforça a importância da base de dados LILACS para a produção da América Latina. A investigação apontou uma maior publicação no período de 2006 a 2012 acerca do tema com os descritores escolhidos, especialmente no início da primeira década do século XXI. Tal achado pode ser corroborado pelo aumento de pesquisadores que se interessam no estudo do idoso e suas interfaces, dado o crescimento populacional mundial, 
que causam alterações demográficas e epidemiológicas ${ }^{(3-}$ 4).

O aumento no número de estudos que abordam a percepção do cuidador também cabe relevância, uma vez que essas pessoas são as que mais tempo permanecem com o idoso ${ }^{(38)}$, com indicativos de redirecionamento das políticas públicas e sociais extensiva às famílias cuidadoras $^{(41)}$. Fato corroborado ao se evidenciar que há associação estatística entre o APGAR de Família e as variáveis composição familiar, sintomas depressivos e alterações cognitivas ${ }^{(42)}$.

A realidade da vivência familiar do idoso é um processo em mudança na sociedade mundial e requer maior atenção, pois reflete alterações sociais complexas, que requerem métodos de pesquisa que, possibilitem a análise de indicadores capazes de mensurar quantitativamente e compreender a dimensão qualitativa do objeto(43).

Quanto à análise do nível de evidência, os achados sinalizam estudos predominantemente do nível IV, o que revela ausência de pesquisas com abordagens e desenhos metodológicos diversificados, que ampliem o conhecimento acerca deste objeto de estudo que é complexo.

Entre os descritores utilizados na pesquisa, os que demonstraram maior presença foram idoso(23,32-36,38-39), família e relações familiares ${ }^{(23,31,34) \text {, }}$ cuidado/cuidador ${ }^{(35,38,41)}$, acrescidos de descritores controlados específicos relacionados ao tema do artigo em questão, como demência/alterações $\operatorname{cognitivas}^{(23,37,40)}$, ambiente familiar(36), depressão(32), IAM(32), osteoartrite $^{(40)}$. Houve também a identificação de um material sem explicitação de DeCs/Mesh no texto publicado(24).

Os autores dos artigos foram profissionais das diversas áreas da saúde, com maior predominância da enfermagem. O que reforça sua fácil aplicação e leitura, por diversos profissionais, especialmente aqueles que atuam na Atenção Primária (AP) e trabalha com idosos e suas famílias na identificação precoce da disfuncionalidade familiar, promoção da funcionalidade, independência funcional e qualidade de vida(33-34).

A avaliação funcional por meio do APGAR de Família é preconizada pela Política Nacional de Saúde da Pessoa Idosa na assistência à saúde do idoso na $A P^{(16)}$. Por se tratar de avaliação objetiva e sistematizada do nível de função familiar na visão do respondente, além de reconhecer rapidamente a disfuncionalidade familiar, de fácil interpretação, baixo custo ${ }^{(16)}$ e alta confiabilidade $^{(23,31)}$.
O índex, nessa perspectiva, por viabilizar compreensão satisfatória da relação familial demonstra ser adequado para o planejamento assistencial ao idoso ${ }^{(35)}$, pois direciona ações interventivas e assertivas no cuidado ao mesmo. Compreender o contexto familiar favorece o atendimento domiciliar, facilita a identificação das reais necessidades de saúde, além de apontar as fragilidades na atenção, antecedendo as ações dos profissionais da área da saúde, sobretudo, os enfermeiros da $\operatorname{AP}^{(14,16,23)}$.

Outro achado relevante quanto à avaliação da FF foi sua aplicação em idosos inseridos na comunidade. Os estudos caracterizam a diversidade em que o índex pode ser utilizado ao ser aplicável em idosos com capacidade funcional comprometida(35-36), idosos independentes, com alterações cognitivas em contextos de alta vulnerabilidade social ${ }^{(23,39)}$ e portadores de agravos específicas como Infarto Agudo do Miocárdio (IAM) e depressão(32).

O teste do APGAR de Família permitiu mensurar qualitativamente a compreensão da dinâmica do funcionamento familiar sob a ótica do idoso, a escolha do cuidador na velhice de idosos independentes ${ }^{(34)}$, dependentes e com alterações cognitivas ${ }^{(35-36,39)}$, apoio aos cuidadores de idosos quanto à sobrecarga de trabalho(37-38,41), além de momento oportuno para se pensar sobre as relações familiares ${ }^{(34)}$ e, número de moradores que residem no domicilio do idoso(39-41).

Sendo assim, a utilização do APGAR de Família permite representar o fenômeno da dinâmica familiar e, esta é de vital importância no processo de valoração do cuidador $^{(24,37,41)}$ e idosos dependentes, com vistas ao planejamento e abordagem do cuidado integralizado ${ }^{(37,39)}$.

Todos os estudos desta RI foram convergentes no sentido de recomendarem o índex APGAR de Família na avaliação da funcionalidade familiar. Conhecer a dinâmica familiar nos domínios avaliados em relação à adaptação, companheirismo, desenvolvimento, afetividade e capacidade resolutiva suscitarão em ações assertivas junto a esta população, uma vez que a satisfação do sujeito índice em relação à sua família contribuirá significativamente na "manutenção da integridade física e psicológica do indivíduo"(36).

Por ser auto aplicável em diferentes momentos no curso da vida do idoso e sua família, permite acompanhar o processo de relações intrafamiliares. Mesmo porque a relação familiar é dinâmica e sofre alterações a todo instante ${ }^{(34-35)}$.

O APGAR de Família também é um instrumento que revela as escolhas ou a condição que conduziu ao papel de cuidador, que por sua vez, sofre diversas variações e 
determinações no decorrer do tempo. Um determinante é o predomínio de mulheres cuidadoras, geralmente as filhas, que já passam pela experiência de cuidar do outro, a composição familiar [famílias multigeracionais] e o relacionamento afetuoso ${ }^{(34,41,44)}$. Esse fenômeno corrobora com a concepção histórica de se atribuir ao gênero feminino a função de cuidador de crianças, doentes e idosos $^{(8)}$.

Em relação ao cuidador, a função familiar mostrou ser um importante preditor da saúde mental do cuidador de pessoas com comprometimento na mobilidade física. Os resultados podem produzir consequências negativas e positivas sobre o cuidador familiar quanto à demanda de cuidados $^{(24,37,41)}$.

$\mathrm{Na}$ avaliação da funcionalidade familial foi evidenciado que existe relação estatisticamente significativa entre os valores corporais, intelectuais, afetivos, individuais, morais, sociais, ecológicos e religiosos com a funcionalidade familiar ${ }^{(31)}$. A prática religiosa, igualmente foi relatada como um recurso de apoio importante para a saúde de idosos, sobretudo, daqueles que sobreviveram a episódio de ataque cardíaco(32). Apresentar escores maiores no APGAR de Família evidenciou melhor qualidade de vida e melhora da dor $^{(40)}$ e também no número de pessoas que residem no domicílio(39).

Ações assertivas evidenciadas nesta RI, orienta conhecer o perfil sociodemográfico, capacidade funcional, função cognitiva, de relacionamento familiar, especialmente, ações que estimulem a reflexão sobre o processo de envelhecimento, cuidado, dependência e velhice. O desenvolvimento de programas de formação e orientação de cuidadores para atuar junto à comunidade também é orientação dos autores pesquisados, no redirecionamento das políticas públicas e sociais favoráveis e extensivas às famílias cuidadores e à rede de apoio de cuidado ao idoso(34,36,41).

Contudo, pesquisas futuras devem se concentrar na satisfação do cuidado recebido e intervenções de cuidadores acerca do funcionamento familiar, a fim de maximizar o funcionamento da família e permitir intervenção precoce ${ }^{(23,39,41,45)}$.

\section{CONCLUSÃO}

A aplicação do APGAR de Família como instrumento de avaliação das relações familiares foi objeto de estudo desta RI, que revelou uma produção de conhecimento de uma tecnologia efetiva no auxilio do planejamento da atenção à saúde do idoso e família na comunidade.

A análise dos artigos demonstrou que o índex de uso multiprofissional pode foi empregado em larga escala para avaliar a concepção do idoso quanto ao ambiente familiar. Além da possibilidade de utilizá-lo em diversas situações de limitações funcionais, alterações cognitivas, vulnerabilidade social ou comorbidade, com aplicação ao longo da vida do idoso.

A autorreferência, uma das características do APGAR de Família, atribui ao idoso à autonomia na avaliação de seu cuidado e, permite ao profissional de saúde (enfermeiro) uma indicação legítima das necessidades de atenção. Interessante notar que tais considerações também se aplicaram cada vez mais em cuidadores de idosos, no que tange a identificação precoce da disfuncionalidade familiar, com vistas a um planejamento de cuidado integral do idoso. O propósito é desenvolver estratégias de atenção domiciliar efetiva às reais necessidades do idoso, do cuidador e de sua família.

Também reforçou as orientações do Ministério da Saúde em aplicar o índex com idosos na família e na comunidade. Destaca-se então, sua viabilidade multidisciplinar no trabalho das equipes de saúde na atenção domiciliar.

Entretanto, uma lacuna na produção de conhecimento foi revelada ao verificar-se escassez da publicação e indexação em base de dados, tendo em vista que no período de janeiro de 2000 a setembro de 2013, foram selecionados treze artigos que atenderam aos critérios de inclusão.

As limitações do estudo compreendeu no fato do acrônimo APGAR de Família não ser um descritor controlado e a dificuldade no acesso a artigos que necessitam de autorização institucional para visualização.

Por fim, considerando o nível de evidência dos estudos, advindos de orientações metodológicas descritivas, sugere-se que pesquisas com delineamento metodológico de evidências com a utilização do índex APGAR de Família sejam mais fortes sejam realizadas, pois um objeto de estudo tão complexo, em uma realidade em transformação, que é o envelhecimento da população mundial, necessita de robusta produção de conhecimento.

Enferm. [Internet]. 2008[cited 2011 ago 13];17(1):72-80. Available from: http://www.scielo.br/pdf/tce/v17n1/08.pdf 2. Teixeira SM, Rodrigues VS. Modelos de família entre idosos: famílias restritas ou extensas? Rev. Bras. Geriatr. Gerontol 
[Internet]. 2009[cited 2011 ago 13]; 12(2):239-54.Available from: http://www.crdeunati.uerj.br/img_tse/v12n2/pdf/art_7.pdf

3. Lebrão ML. O envelhecimento no Brasil: aspecto da transição demográfica e epidemiológica. Saúde Coletiva [Internet]. 2007[cited 2011 ago 13];4(17):135-40. Available from: http://redalyc.uaemex.mx/src/inicio/ArtPdfRed.jsp?iCve $=842017$ $\underline{03}$

4. Veras R. Envelhecimento populacional contemporâneo: demandas, desafios e inovações. Rev. Saúde Pública [Internet]. 2009[cited 2011 dez 17];43(3): 548-554. Available from: http://www.scielosp.org/pdf/rsp/2009nahead/224.pdf 5. Ramos LR. Fatores determinantes do envelhecimento saudável em idosos residentes em centro urbano: Projeto Epidoso, São Paulo. Cad. Saúde Pública [Internet]. 2003[cited $2011 \mathrm{dez}$ 17];19(3):793-798. Available from: http://www.scielosp.org/pdf/csp/v19n3/15882.pdf

6. Mazza MMPR, Lefèvre. Cuidar em família: análise da representação social da relação do cuidador familiar com o idoso. Rev. Bras Cresc Desenv Hum [Internet]. 2005[cited $2011 \mathrm{dez}$ 17];15(1):01-10. Available from:

http://www.revistasusp.sibi.usp.br/pdf/rbcdh/v15n1/02.pdf 7. Ministério da Saúde. Secretaria de Atenção à Saúde. Departamento de Ações Programáticas e Estratégicas. Atenção à saúde da pessoa idosa e envelhecimento. Área técnica saúde do idoso. Série B. Textos Básicos de Saúde. Brasília: Ministério da Saúde; 2010.

8. Moragas RM. Gerontologia social. Envelhecimento de qualidade de vida. 3rd rev. ed. São Paulo: Paulinas; 2010. 343 p. 9. Alcântara AO. O velho e a família. In: Alcântara AO. Velhos institucionalizados e família. Entre abafos e desabafos. 2nd ed. Campinas: Alínea; 2009. p. 21-30.

10. Mendes MRSSB, Gusmão JL, Faro ACM, Leite RCBO. The social situation of elderly in Brazil: a brief consideration. Acta Paul Enferm [Internet]. 2005[cited $2011 \mathrm{dez} 17$ ];18(4):422-6. Available from:

http://www.scielo.br/pdf/ape/v18n4/a11v18n4.pdf 11. Caldas CP. Envelhecimento com dependência: responsabilidades e demandas da família. Cad. Saúde Pública [Internet]. 2003[cited $2011 \mathrm{dez} 17$ ]; 19(3): 773-781. Available from: http://www.scielo.br/pdf/csp/v19n3/15880.pdf 12. World Health Organization. Envelhecimento ativo: uma política de saúde. Trad. Suzana Gontijo. Brasília: Ministério da Saúde; 2005.

13. Smilkstein G. The family APGAR: a proposal for a family function test and its use by physicians. J Fam Practice [Internet]. 1978 [cited 2011 dez 17];6(6):1231-1239. Available from: http://www.ncbi.nlm.nih.gov/pubmed/660126.

14. Duarte YAO. Família: rede de suporte ou fator estressor. A ótica de idosos e cuidadores familiares. [these] São Paulo (São Paulo): Universidade de São Paulo -Escola de Enfermagem; 2001. 196p.

15. Santi, PMH. La familia funcional y disfuncional, un indicador de salud. Rev Cubana Med Gen Integr [Internet].1997 [cited $2011 \mathrm{dez} 17] ; 13(6)$. Available from: http://bvs.sld.cu/revistas/mgi/vol13 6 97/mgi13697.htm 16. Ministério da Saúde. Secretaria de Atenção à Saúde. Departamento de Atenção Básica. Envelhecimento e saúde da pessoa idosa. Brasília: Ministério da Saúde; 2007. 17. Ok-Soo K, Sung-Hee B. The relationships among loneliness, social support, and family function in elderly Korean. J Korean Acad Nurs [Internet]. 2003 [cited 2011 dez 17]; 33(3):425-432. Available from: http://www.ncbi.nlm.nih.gov/pubmed/15314441 18. Ocampo JM, Romero N, Saa HA, Herreira JA, Reyes-Ortiz CA Prevalencia de las prácticas religiosas, disfunción familiar, soporte social y síntomas depresivos en adultos mayores. Cali, Colombia-2001. Colombia Médica [Internet]. 2006 [cited 2011 dez 17];37(2 Suppl 1):S26-30. Available from:

http://www.bioline.org.br/pdf?rc06033

19. Martini AM, Sousa FGM, Gonçalves APF, Lopes, MLH. Estrutura e funcionalidade de famílias de adolescentes em tratamento Hemodialítico. Rev. Eletr. Enf.[Internet].2007 [cited 2011 ago 13]; 9(2): 329-343. Available from: http://www.revistas.ufg.br/index.php/fen/article/view/7163
20. Sun TH, Sun L, Wu HY, Zhang Zk, Wang B, Yu C, Cao HY. Loneliness, social support and family function of people living with HIV/AIDS in Anhui rural area, China. Int J STD AIDS [Internet]. 2009 [cited $2011 \mathrm{dez} 17$ ]; 20(4):255-8. Available from: http://ijsa.rsmjournals.com/content/20/4/255. full 21. Chiou CJ, Chang HY, Chen IP, Wang HH. Social support and caregiving circumstances as predictors of caregiver burden in Taiwan. Arch Gerontol Geriat [Internet]. 2009 [cited 2011 ago 13]; 48(3):419-24. Avalilable from:

http://www.ncbi.nlm.nih.gov/pubmed/18602706

22. Abilés V, Rodríguez-Ruiz S, Abilés J, Mellado CA, García A, Pérez de La Cruz A, Fernández-Santaella MC. Psychological Characteristics of Morbidly Obese Candidates for Bariatric Surgery. Obes Surg [Internet]. 2010 [cited 2011 ago 13]; 20:161-167. Available from:

http://www.ncbi.nlm.nih.gov/pubmed/18958537

23. Santos AA, Pavarini SCI, Barham EJ. Percepção de idosos pobres com alterações cognitivas sobre funcionalidade familiar. Texto contexto - enferm. [Internet]. 2011[cited 2011 dez 17]; 20(1),102-110. Available from:

http://www.scielo.br/pdf/tce/v20n1/12.pdf

24. Rodríguez-Sánchez $E$, Pérez-Peñaranda $A$, Losada-Baltar $A$, Pérez-Arechaederra D, Gómez-Marcos MÁ, Patino-Alonso M, García-Ortiz L. Relationships between quality of life and family function in caregiver. BMC Family Practice [Internet]. 2011 [cited 2011 dez 17]; 12:19. Available from:

http://www.biomedcentral.com/1471-2296/12/19

25. Tabares OD, Quintana MLS, Capote MG. El apgar familiar en ancianos conviventes. Rev Cubana Med Gen Integr [Internet].1998 [cited $2011 \mathrm{dez} 19] ; 14(6): 548-53$. Available from: http://bvs.sld.cu/revistas/mgi/vol14_6_98/mgi07698.pdf 26. Ganong LH. Integrative reviews of nursing research. Research in Nursing \& Health. 1987 10:1-11.

27. Whittemore $R$, Knafl K. The integrative review: updated methodology. Journal of Advanced Nursing. [Internet].2005 [cited 2012 jun 03]; 52(5):546-53. Available from: http://users.phhp.ufl.edu/rbauer/EBPP/whittemore_knafl_05.pdf 28. Galvão CM, Mendes KDS, Silveira RCCP. Revisão integrativa: método de revisão para sintetizar as evidências disponíveis na literatura. In: Brevidelli MM, Sertório SCM, editors. TCCTrabalho de Conclusão de Curso: guia prático para docentes e alunos da área da saúde. 4 ed. São Paulo: Iátria; 2010. p.105126.

29. Ursi ES. Prevenção de lesões de pele no perioperatório: revisão integrativa da literatura. [dissertation]. Ribeirão Preto (São Paulo): Universidade de São Paulo - Escola de Enfermagem de Ribeirão Preto; 2005. 128p.

30. Stetler CB, Morsi D, Ruckis, Broughton S, Corrigan B,

Fitzgerald J, et al. Utilization-focused integrative reviews in a nursing service. Appl Nurs Res [Internet]. 1998[cited 2011 dez 17];11(4):195-206. Available from:

http://www.ncbi.nlm.nih.gov/pubmed/9852663

31. Ferrer M. Valores y funcionabilidad familiar en habitantes del sector San Jose Coro-Falcón. MedFam Caracas. 2003 jul-dec; 11(2):20-7.

32. Reyes CA, Hincapié M, Herrera MJA, Moyano PA. Factores de estrés y apoyo psicosocial en pacientes con infarto agudo de miocardio. Cali, 2001-2002. Colomb Med [Internet] 2004 [citado $2011 \mathrm{dez} 17]$; 35(4):199-204. Available from: http://www.doaj.org/doaj?func=abstract\&id $=112064$ 33. Zavala GM, Vidal GD, Castro SM, Quiroga P, Gonzalo KP. Funcionamiento social del adulto mayor. Cienc. Enferm [Internet]. 2006 [cited $2011 \mathrm{dez} 17$ ];12(2):53-62. Available from: http://www.scielo.cl/pdf/cienf/v12n2/art07.pdf 34. Pavarini SCI, Tonon FL, Silva JMC, Mendiondo MSZ, Barham EJ, Filizola CLA. Quem irá empurrar minha cadeira de rodas? A escolha do cuidador familiar do idoso. Rev Eletr Enferm [Internet]. 2006 [citado $2011 \mathrm{dez} 17$ ]; 8(3). Available from: http://www.fen.ufg.br/revista/revista8 3/v8n3a03.htm. 35. Torres GV, Reis LA, Reis LA, Fernandes MH, Alves GS, Sampaio LS, Mascarenhas CHM. Funcionalidade familiar de idosos dependentes residentes em domicílios. Aval Psicol [Internet]. 2009 [cited $2011 \mathrm{dez} 17$ ]; 8(3):415-23. Available from: http://pepsic.bvsalud.org/pdf/avp/v8n3/v8n3a13.pdf 
36. Torres GV, Reis LA, Reis LA, Fernandes MH, Xavier TT. Relação entre funcionalidade familiar e capacidade funcional de idosos dependentes no município de Jequié (BA). Rev. baiana saúde pública [Internet]. 2010 [cited $2011 \mathrm{dez} 17$ ];34(1), p. 1930. Available from: http://files.bvs.br/upload/S/01000233/2010/v34n1/a1398.pdf

37. Perez-Penaranda A, Garcia Ortiz L, Rodriguez Sanchez E, Losada Baltar A, Porras Santos N, Gomez Marcos MA: Family function and the mental health of the caregiver of dependent relatives. Aten Primaria [Internet]. 2009 [cited 2011 dez 17]; 41(11):621-628. Available from:

http://www.ncbi.nlm.nih.gov/pubmed/19497641

38. Ocampo JM, Herrera JA, Torres P, Rodríguez JA, Loboa

L,García CA. Sobrecarga asociada con el cuidado de ancianos dependientes. Colombia Med [Internet]. 2007 [cited 2013 out 04] ; 38(1):40-46. Available from:

http://www.scielo.org.co/pdf/cm/v38n1/v38n1a05.pdf

39. Santos AA; Pavarini SCI. Family functionality regarding the elderly with cognitive impairments: the caretaker's perception. Rev. esc. enferm. USP [Internet]. 2012 [cited 2013 out 04]; 46(5): 1141-1147. Available from:

http://www.scielo.br/pdf/reeusp/v46n5/15.pdf

40. Lim AT, Manching I, Penserga EG. Correlation between

Family APGAR scores and health-related quality of life of Filipino

elderly patients with knee osteoarthritis. Int J Rheum

Dis.[Internet]. 2012 [cited 2013 out 04]; 15(4):407-4013.

Available from: http://www.ncbi.nlm.nih.gov/pubmed/22898221

41. Gonçalves LTH; Leite MT; Hildebrandt LM; Bisogno SC;

Biasuz S; Falcade BL. Convívio e cuidado familiar na quarta

idade: qualidade de vida de idosos e seus cuidadores. Rev. bras. geriatr. gerontol. [Internet]. 2013 [cited 2013 out 04];

16(2):315-325. Available from:

http://www.scielo.br/pdf/rbgg/v16n2/11.pdf

42. Oliveira SC, Pavarini SCI, Orlandi FS, Mediondo MSZ. Family

functionality: a study of Brazilian institucionalized elerly

individuals. Arch. Gerontolol. Geriatr [Internet].2013 [cited 2013

out 01$] ; 57(3)$ : pages 7. Available from:

http://www.sciencedirect.com/science/article/pii/S01674943130

$\underline{01106}$

43. Minayo MCS. Mudança: conceito-chave para intervenções sociais e para avaliação de programas. In: Minayo MCS, Assis SG, Souza ER (orgs). Avaliação por triangulação de métodos: abordagem de programas sociais. Rio de Janeiro: Editora Fiocruz, 2005, p.53-70.

44. Uesugui HM, Fagundes DS, Pinho DLM. Profile and degree of dependency of the elderly and overload of their caregivers. Acta Paul Enferm [Internet]. 2011 [cited $2011 \mathrm{dez}$ 17]; 24(5):685698. Available from:

http://www.scielo.br/pdf/ape/v24n5/en_15v24n5.pdf

45. Heru $A$, Ryan CE, Iqbal A: Family functioning in the caregivers of patients with dementia. J Geriatr Psychiatry [Internet]. 2004 [cited 2011 dez 17]; 19:533-537. Available from: http://www.ncbi.nlm.nih.gov/pubmed/15211531

Artigo recebido em 09/02/12.

Aprovado para publicação em 16/09/13.

Artigo publicado em 31/03/2014. 